ONCOGENESIS

\section{A life or death situation}

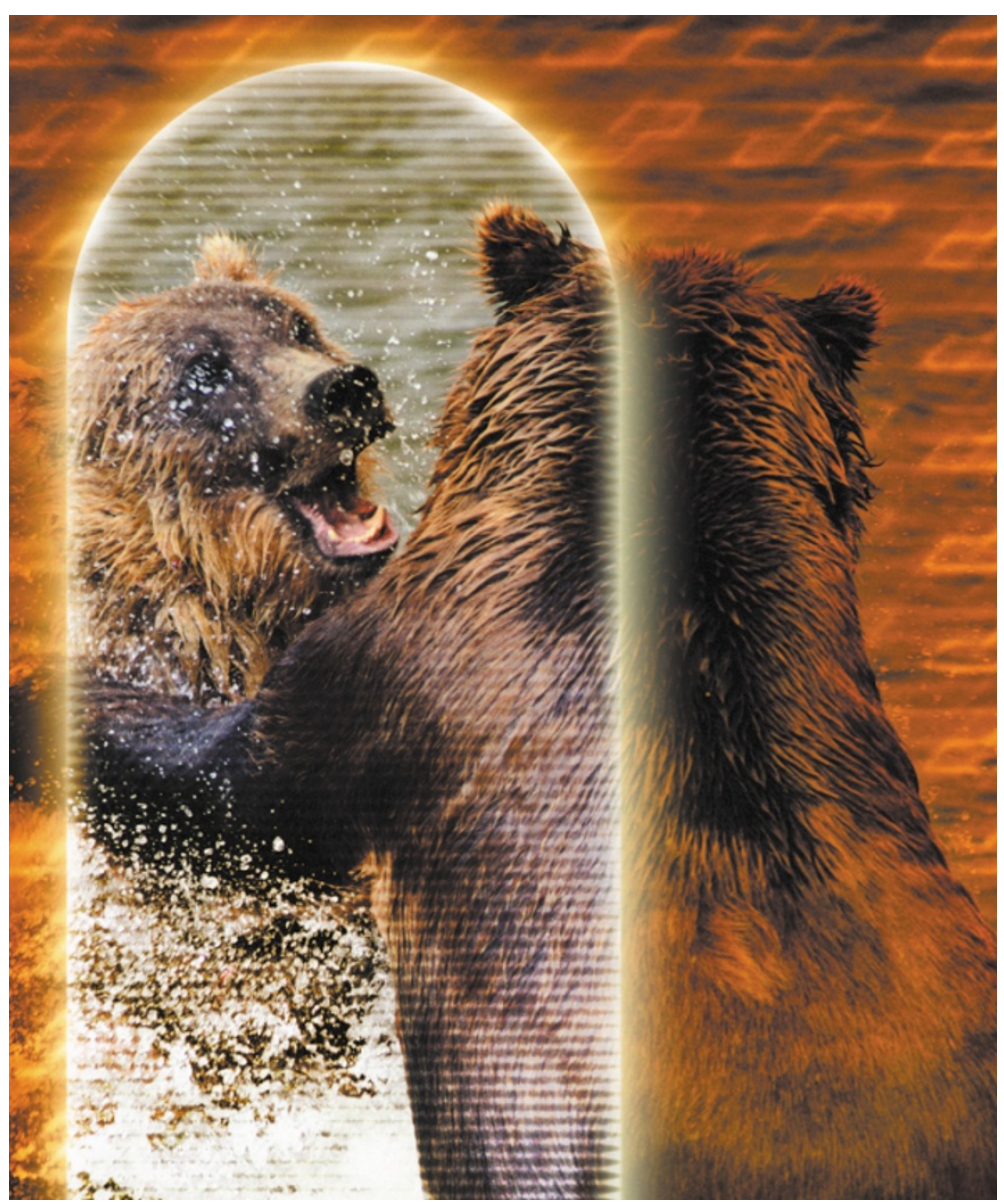

The oncogene c-Myc controls the fine line between life and death, as it can induce both cell proliferation and apoptosis. But whether c-Myc-induced cell death can actually restrain tumour growth has remained undetermined. Stella Pelengaris et al. have now shown that c-Myc-induced apoptosis can indeed prevent tumour formation, and that switching off apoptosis allows the tumorigenic capability of c-Myc to proceed unchecked.

A switchable c-Myc - in which the gene is fused to the 4-hydroxytamoxifen (4-OHT)-responsive oestrogen receptor $\left(c-M y c E R^{T A M}\right)$, so the protein is activated following the intraperitoneal administration of 4OHT - was specifically targeted to pancreatic $\beta$-cells in mice using the pIns insulin promoter. Induction of c-Myc initially resulted in cell proliferation, but this was accompanied by a faster rate of apoptosis, such that the net effect was $\beta$-cell ablation and hyperglycaemia caused by loss of insulin-producing cells. Interestingly, when 4-OHT was withdrawn, which switched off c-Myc, pancreatic islets rapidly regenerated and blood glucose levels returned to normal.

If apoptosis overpowers cell proliferation, inhibiting apoptosis should allow proliferation to proceed unchecked. Expressing the apoptosis inhibitor $B c l-x_{L}$ under the control of the rat insulin promoter (RIP7) allowed this hypothesis to be tested. pIns-c-MycER $R^{\text {TAM }} / R I P-B c l-x_{L}$ transgenic mice had normal pancreatic islet formation until 4-OHT was added, at which point proliferation was induced throughout the pancreatic $\beta$-cells. As apoptosis was inhibited by Bcl- $\mathrm{x}_{\mathrm{L}}$, this resulted in hyperplasia within 7 days.

But can deregulated expression of c-Myc induce tumour formation, which is thought to require the cumulative effect of multiple mutations? Pancreatic $\beta$-cells in pIns-c$M y c E R^{T A M} / R I P-B c l-x_{L}$ mice not only hyperproliferated, but also underwent de-differentiation - as seen by the reduced production of insulin - and extensive angiogenesis. Expression of the intercellular adhesion molecule E-cadherin was lost as well, which is a prerequisite for loss of cell-cell contacts and invasion. c-Myc therefore seems to be able to directly induce several of the hallmarks of cancer. Two weeks after induction of $\mathrm{c}-\mathrm{Myc}$ expression, pIns-c-MycER $R^{\text {TAM }} / R I P-B c l-x_{L}$ mice had developed pancreatic tumours, and by 8 weeks the tumours were large and vascularized, with sites of local invasion in local blood vessels and draining lymph nodes.

So, expression of a single oncogene - c-Myc - is sufficient to induce several steps of carcinogenesis, as long as its innate apoptotic activity
CELLULAR MICROBIOLOGY

\section{An inhospitable host}

What makes a host inhospitable? Is it when they don't offer you a drink when you arrive at their home? Clearly, this is not what makes a host inhospitable to Shigella, although the factor that makes host neutrophils unwelcoming has now been identified. In Nature, Zychlinsky and colleagues show that neutrophil elastase (NE) attacks Shigella virulence factors, making this bacterium extremely unwelcome.

In macrophages and epithelial cells, Shigella escape to the cytoplasm from phagosomes, where they would otherwise be destroyed. Neutrophils, however, trap bacterial cells inside phagosomes. So what is the difference between these host cells?

Zychlinsky and co-workers investigated the effect of human neutrophil extract enriched in granule proteins (hNEGP) on Shigella. High concentrations of hNEGP killed Shigella, and sublethal concentrations specifically decreased the levels of the secreted type III virulence factors IpaA, IpaB and $\mathrm{IpaC}$, which are required for invasion of the host's cytoplasm. The level of the outermembrane virulence protein IcsA - which is required for intracellular movement - was also specifically decreased.

Using chemical inhibitors, the authors identified $\mathrm{NE}$ as the perpetrator of this degradation, and showed that NE degrades virulence factors at 1,000 -fold lower concentrations than those required to degrade other bacterial proteins. Zychlinsky and colleagues further illustrated the specificity of NE by showing that virulence factors were cleaved in preference to, for example, virulence-associated proteins, even though the latter were present in larger amounts in the cultures used.

In intact neutrophils, the authors found that
IpaA, IpaB and IcsA were specifically degraded on Shigella infection, an event that did not occur in cells treated with an NEspecific inhibitor. So what happens to Shigella when NE is absent in vivo? In both chemically inhibited human neutrophils and NE-null mice, the authors found that bacterial cells could escape to the cytoplasm, and that this escape correlated with increased Shigella survival. By contrast, Shigella remained in the phagosomes of wild-type cells.

The authors also found that NE targets the virulence proteins of two other pathogens Salmonella and Yersinia - and future work will investigate the NE susceptibility of other bacteria. This work establishes NE as the first neutrophil factor that targets bacterial virulence proteins. So, now we know what makes neutrophils such inhospitable hosts. Rachel Smallridge

(2) References and links ORIGINAL RESEARCH PAPER Weinrauch, $Y$. et $a l$. Neutrophil elastase targets virulence factors of enterobacteria.
NerinAL Nature 417, 91-94 (2002) 
is curtailed; but is it also required to maintain the tumours once they have formed? Switching off c-Myc, 14 days after its induction, resulted in a reversal of the tumorigenic process: $\beta$-cells exited the cell cycle, E-cadherin was re-expressed and cells reestablished cell-cell contacts, and endothelial cells and $\beta$-cells apoptosed. Even mice that had expressed c-Myc for 8 weeks, with extensive tumours that had invaded into lymph nodes, made a full recovery following c-Myc deactivation.

These results challenge the paradigm that carcinogenesis is a multistep process that requires many mutations, and indicate that, instead, it can be driven by deregulated expression of a single growth-deregulating oncogene, provided apoptosis is suppressed. If this is found to be true for other commonly mutated oncogenes, new cancer therapeutics should aim to inhibit these few crucial molecular targets.

Emma Greenwood, Associate Editor, Nature Reviews Cancer (2) References and links ORIGINAL RESEARCH PAPER Pelengaris, $S$. et al. Suppression of Myc-induced apoptosis in $\beta$-cells exposes multiple oncogenic properties of Myc and triggers carcinogenic progression. Cell 109, 321-334 (2002)

WEB SITES

Encyclopedia of Life Sciences: http://www.els.net

Apoptosis: molecular mechanisms Gerard Evan's laboratory: http://cc.ucsf.edu/evan/

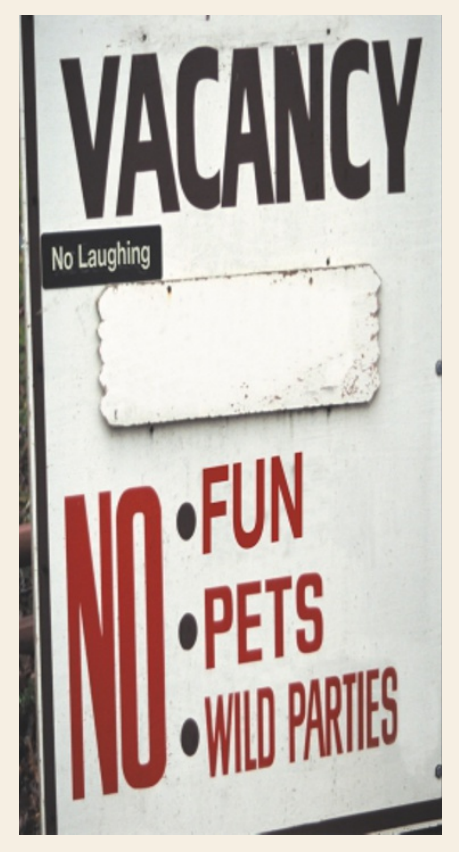

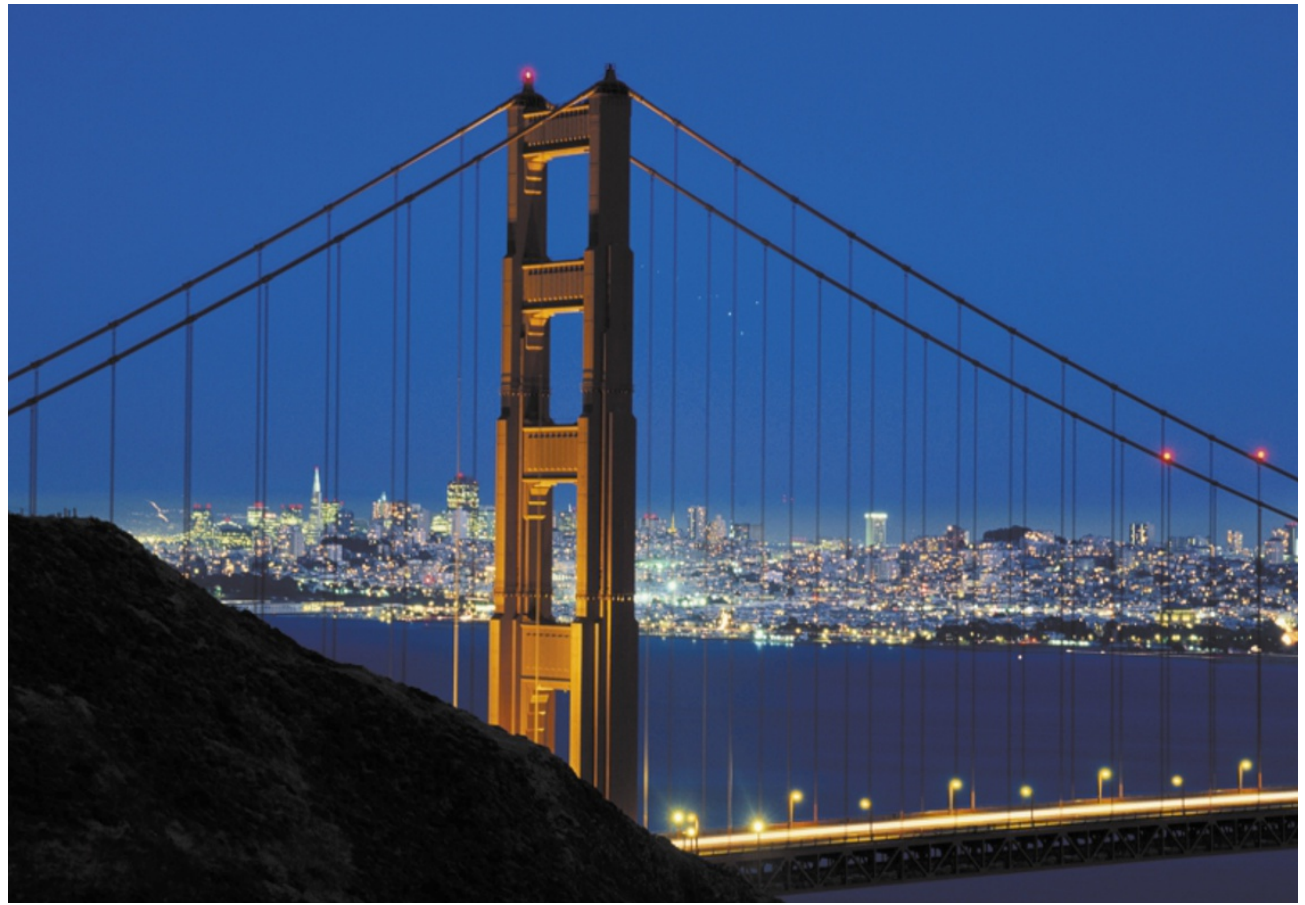

UBIQUITYLATION

\section{A supporting role}

Thousands of chemical modifications - such as ubiquitylation - occur after proteins have been synthesized. One function of ubiquitylation is to target proteins for degradation by the $26 \mathrm{~S}$ proteasome. SCF (Skp1-Cullin-F-box protein) and SCF-like complexes are the largest family of ubiquitin-dependent ligases - or E3s - that mediate one step of ubiquitylation, and Pavletich and colleagues now describe the 3.2- $\AA$ crystal structure of the Cul1-Rbx1-Skp1-F-box ${ }^{\text {Skp2 }}$ SCF complex in Nature.

E3s act at the last step of a process that involves ubiquitin-activating (E1) and ubiquitin-conjugating (E2) enzymes. They have an important role in conferring specificity on the ubiquitylation pathway, as they bind to both an E2 and a protein substrate to mediate the transfer of ubiquitin between them.

Pavletich and co-workers found that the Cul1-Rbx1-Skp1-F-box ${ }^{\text {Skp2 }}$ SCF complex has an elongated structure, with Rbx1 (a RING finger protein that is an essential component of SCF) and Skp1-F-box ${ }^{\text {Skp2 }}$ - the protein substrate-recognition complex - located at opposite ends of the complex. They showed that this arrangement is organized by Cul1 - an elongated protein with a long stalk (amino-terminal helical region or NTD) and a carboxy-terminal globular domain (CTD). Cull acts as a scaffold, making contacts with all of the other SCF subunits.

The authors found that the Cull NTD is made up of three cullin-repeat motifs, which form a long arc shape, and that the amino-terminal tip of the first repeat binds Skp1-F-box ${ }^{\text {skp2 }}$. They also established that the Cull CTD binds Rbxl through an intermolecular $\beta$-sheet to form the region that recruits E2s.
Pavletich and colleageus observed no flexible linkages in the Cul1-Rbx1-Skp1-F-box ${ }^{\text {Skp2 }}$ complex, so they made a Cul1-mutant construct to test the importance of this rigid architecture. In this mutant, the NTD-CTD interface was disrupted and these domains were connected by a flexible linker. Although this construct could still bind to its protein substrate in the presence of the necessary substrate-recognition proteins, and could polymerize ubiquitin independently of substrate, the authors found that it could not ubiquitylate its substrate in vitro. They took this to indicate that the rigidity of the Cull scaffold, and of the entire SCF, is important for E3 activity.

The SCF structure was used by the authors to make a model of an SCF-E2 complex, which showed that the protein substrate and the E2 would be located on one side of the SCF complex. This architecture led them to propose that Cull evolved its long stalk to keep the substrate binding and catalytic activities separate, and to accommodate substrates of different sizes that have varying spaces between their prospective ubiquitylation site and their SCF-binding motif.

They further speculate that this type of E3 might facilitate the transfer of ubiquitin by positioning the protein substrate in a way that optimizes its presentation to the E2. Although the extent to which this positioning occurs is unclear, they propose that, as no sequence or structural motif has yet been identified for a ubiquitylation site, the spatial constraints that are imposed by E3s might have an important role in determining this specificity.

Rachel Smallridge

\section{2) References and links}

ORIGINAL RESEARCH PAPER Pavletich, N. P. et al. Structure of the Cul1-Rbx1-Skp1-F-box ${ }^{\text {Skp2 }}$ SCF ubiquitin ligase complex.

Nature 416, 703-709 (2002)

FURTHER READING Weissman, A. M. Ubiquitin and proteasomes: themes and variations on ubiquitylation. Nature Rev. Mol. Cell Biol. 2, 169-178 (2001)

WEB SITES

Encyclopedia of Life Sciences: http://www.els.net

Ubiquitin pathway

Nikola Pavletich's laboratory:

http://www.ski.edu/lab_homepage.cfm?lab=144 